Research Article

\title{
Detection of Illegal Movement in Radioactive Material Transportation Security Systems
}

\author{
Tiejun Zeng $\mathbb{D}^{1,2}$ Xiaohua Yang $\mathbb{D}^{1,3,4}$ Yaping Wan $\mathbb{D}^{3,4}$ Panpan Jiang $\mathbb{D}^{1,2}$ \\ and Liu Zhenghai $\mathbb{1}^{4}$ \\ ${ }^{1}$ School of Nuclear Science and Technology, University of South China, Hengyang 421001, China \\ ${ }^{2}$ School of Electrical Engineering, University of South China, Hengyang 421001, China \\ ${ }^{3}$ CNNC Key Laboratory on High Trusted Computing, Hengyang 421001, China \\ ${ }^{4}$ School of Computer, University of South China, Hengyang 421001, China \\ Correspondence should be addressed to Tiejun Zeng; ztj182002@163.com and Xiaohua Yang; xiaohua1963@usc.edu.cn
}

Received 30 December 2020; Revised 14 May 2021; Accepted 28 August 2021; Published 10 September 2021

Academic Editor: Michel Giot

Copyright $\odot 2021$ Tiejun Zeng et al. This is an open access article distributed under the Creative Commons Attribution License, which permits unrestricted use, distribution, and reproduction in any medium, provided the original work is properly cited.

The loss and theft of radioactive material in transport can be attributed to the illegal movement. In order to distinguish it from the movement caused by the turbulence of the transportation vehicle, this paper proposes the criterion of "illegal movement" as the movement of radioactive materials outside the transportation compartment. Since the interior of the compartment is generally a metal environment, this paper proposes wireless signal strength data as a sensing method. The wireless signal strength data is filtered and converted into distance data. We construct a spatial triangle perpendicular to the top and sides of the compartment based on the distance data. When the radioactive material is inside the compartment, the angle between its corresponding point and the top plane of the compartment is less than $90^{\circ}$. Once it moves out of the compartment, the angle will be greater than $90^{\circ}$. Based on this, a sensing method of "illegal movement" based on spatial triangles is proposed. The simulation research shows that the scheme proposed in this paper is feasible.

\section{Introduction}

The transportation of radioactive material is characterized by long path, complex road condition, and limited response power [1]. In addition, the security of radioactive materials during transportation is more fragile than during storage [2]. Loss of radioactive material during transportation occurred in several countries in the last decade. For example, a package containing two A424-9 Ir-192 industrial radiographic sealed sources was lost during transportation on October 9, 2020. Tracking indicates the last known package location was a common carrier hub in Memphis, TN [3]. IAEA guidance document on transport security-No.9 G was published in July 2020 [4], in which the per package approach for specifying the transport security level is applied. It points out the requirements for the detection of illegal movements. The security system should provide detection of any unauthorized removal of the package at basic transport security level, provide detection of any attempted unauthorized removal of the package at enhanced transport security level, and provide immediate detection of any attempted unauthorized removal of the package at additional security measures.

Since the transport security level can be determined according to the package, we believe that it is necessary to decentralize security measures to the package, not only just the conveyances of transportation. As one of the implementation methods, giving the radioactive material "selfsecurity intelligence" $[5,6]$ (adding intelligent electronic device to the radioactive material container), detect its illegal approach and illegal movement through the connection between a single package (security device) or multiple packages (security device) of radioactive material. We proposed a new vehicle transportation security system based on self-security intelligence functions in literature [1]. This article [1] is mainly aimed at the passive problem of the 
security system during the transportation of radioactive materials and proposes the theory of self-security intelligence, that is, to realize perception, decision-making, and response on the radioactive materials themselves (containers), and put forward the implementation plan on the transportation vehicle. The biggest innovation of this article is to put forward a security concept different from traditional transportation security and propose active protection for the original passive protection.

This article and article [7] both focus on the loss and theft of radioactive materials during transportation. The detection distance of the two follows the order from far to near, and the detection time is from long to short (if the same communication method is used). Based on the illegal movement detection method of failure detection [7], when the individual self-security security device installed on the radioactive material container is fixedly connected to the container itself, it can be considered that the movement of the individual self-security security device is the movement of the radioactive material (specific fixed connection technology not discussed here). When the radioactive material and the transportation compartment can maintain a communication link, regardless of the damage of the device itself, the distance between the two is not far. When the distance between the radioactive material and the transport carriage is too far, causing the communication between the two to be interrupted, the failure detection method can be used to detect at this time.

The detection distance threshold set in this paper is on the side wall of the carriage. Considering the error, it can be detected only by a certain distance beyond the side wall of the carriage. At this time, there is still a considerable distance from the communication failure, so the detection distance is shorter than the article [7], and the alarm time is shorter.

\section{Analysis of Illegal Movement Behavior}

The Regulations on the Road Transportation of Radioactive Materials promulgated by China in 2016 (revised edition) stipulates the requirements for transport vehicles [8]. The approved load of 1 ton or less is a van (as shown in Figures 1 and 2) or a closed truck.

According to Security of Radioactive Material in Transport published by IAEA in 2020 [4], the illegal movement detection functions of the security system are as in Table 1.

Radioactive material (container) will leave the compartment after being stolen or lost, that is, move outside of the compartment. The destruction of the radioactive material (container) will also destroy the self-security device on it. As a result, the self-security device cannot feel its position from the compartment. It can also be considered that it has moved to an infinite distance, also out of the compartment. All of the above movements can be regarded as "illegal movements." During normal road transportation, as shown in Figures 2 and 3, uneven roads cause bumps, and the radioactive materials container will have a certain degree of displacement in the compartment. But this is normal movement. Illegal movement is abnormal movement. In

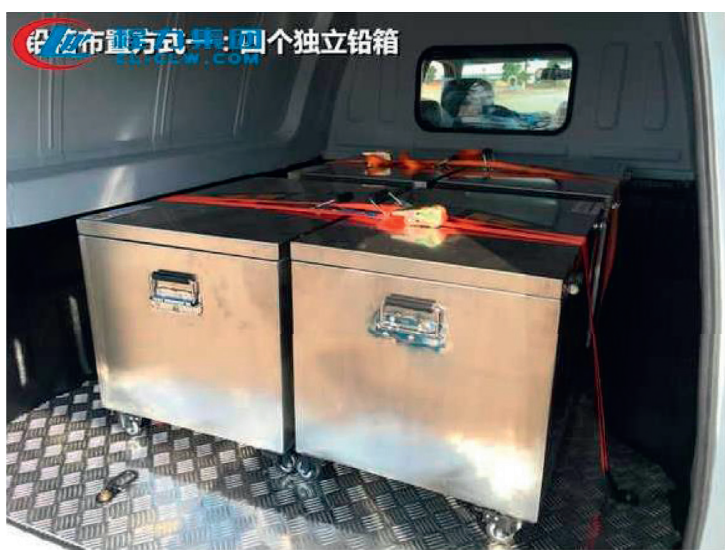

FIGURE 1: Radioactive material carrier 1.

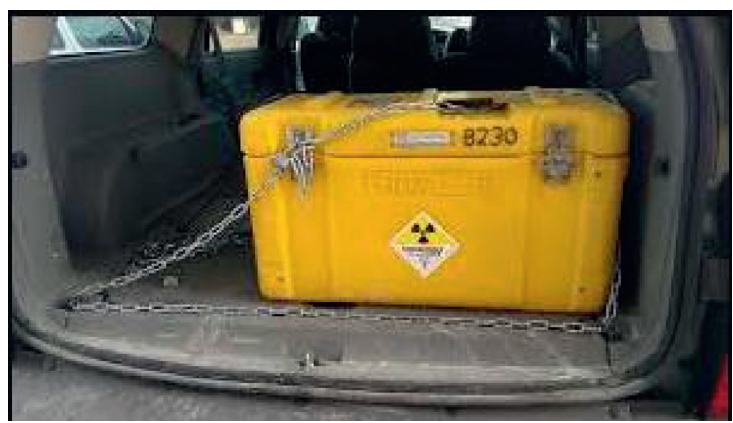

FIgURE 2: Radioactive material carrier 2.

order to distinguish it from normal movement, "illegal movement" here is defined as moving outside of the compartment. Because the transportation compartment is a metal space environment, positioning technologies such as GPS [9, 10] or BeiDou [2] will not receive signals and become invalid.

There are two main types of positioning methods based on wireless signal strength data (RSSI): ranging and nonranging. The positioning method based on ranging first converts the wireless signal strength into the distance between two nodes and then uses related geometric methods, such as the relationship between three sides or triangles for positioning [11]. The positioning method based on nonranging only determines the number of hops between nodes in the network, then estimates the approximate distance of each hop, then multiplies it to get the approximate distance, and then uses geometric relationships to locate [12]. The accuracy of the latter positioning method is lower. In this paper, based on the wireless signal strength (RSSI) data, a space triangle method is proposed to locate the radioactive material in the transport compartment to identify whether it moves outside of the compartment.

\section{Model and Method of Illegal Movement Detection Based on Space Triangle}

Arrange anchor nodes in the transport compartment. The anchor nodes broadcast wireless positioning signals in turn. After receiving the wireless positioning signals, the self- 
TABLE 1: Targets of illegal movement detection function under different transportation security levels.

\begin{tabular}{cccc}
\hline Basic transport security level & $\begin{array}{c}\text { Enhanced transport security } \\
\text { level }\end{array}$ & Additional security measures \\
\hline \multirow{2}{*}{$\begin{array}{c}\text { Detection } \\
\text { Provide detection of any unauthorized removal of } \\
\text { the package }\end{array}$} & $\begin{array}{c}\text { Provide detection of any } \\
\text { attempted } \\
\text { unauthorized removal of the } \\
\text { package }\end{array}$ & $\begin{array}{c}\text { Provide immediate detection of } \\
\text { any attempted unauthorized removal of } \\
\text { the package }\end{array}$ \\
\hline
\end{tabular}

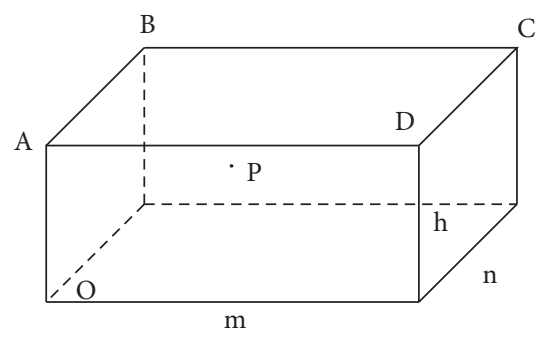

Figure 3: A simple scheme of the different elements of the detection model.

security devices extract the corresponding wireless signal strength and convert it to a spatial distance. Use these distances to construct a spatial triangle and determine whether the self-security device has moved outside of the compartment. This judgment result is sent to the bicycle security platform via the wireless network to provide data support for its alarm decision-making.

3.1. Illegal Movement Detection Model Based on Space Triangle. The four anchor nodes are installed in the four corners of the compartment close to the roof (in order not to affect the wireless communication effect, the antenna is at least $10 \mathrm{~cm}$ away from the roof and the inner wall of the compartment), in a rectangular arrangement, as shown in Figure 4. Point $\mathrm{A}$ is at the front left, and point $\mathrm{B}$ is at the front right. Point $\mathrm{C}$ is at the right rear, and point $D$ is at the left rear. The area of $A B C D$ represents the internal area of the compartment. The radioactive material (container) is placed in a single layer, and the self-security device is installed on the top of the container; particularly, the antenna cannot be blocked. The self-security device $\mathrm{P}$ forms a triangle $\mathrm{ABP}$, $\mathrm{BCP}, \mathrm{CDP}$, and DAP with points $\mathrm{A}, \mathrm{B}, \mathrm{C}$, and D. Due to the different heights of the radioactive materials (containers), point $\mathrm{P}$ and points $\mathrm{A}, \mathrm{B}, \mathrm{C}$, and $\mathrm{D}$ will not be on the same plane. In Figure 3, $m$ represents the length of the compartment, $n$ represents the width of the compartment, $h$ represents the height of the compartment, and point $\mathrm{O}$ represents the lower left corner of the compartment.

When the self-security device $\mathrm{P}$ is located in the compartment, as shown in Figure 4, cross point $\mathrm{P}$ to make a vertical plane with the planes $A B C D$ and $A B O$, cross the line $\mathrm{AB}$ at point $\mathrm{E}$, and cross the line $\mathrm{CD}$ at point $\mathrm{F}$. $\mathrm{PE}$ is perpendicular to $\mathrm{AB}$ in the triangle $\mathrm{PAB}$. $\mathrm{PF}$ is perpendicular to $\mathrm{CD}$ in the triangle PCD. In triangular PEF, the angles $\angle \mathrm{PFE}$ and $\angle \mathrm{PEF}$ are both less than $90^{\circ}$. The cosine law of $\angle \mathrm{PFE}$ is as shown in the following formula:

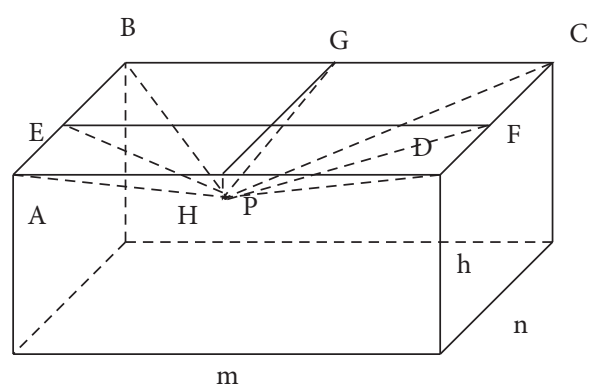

Figure 4: Point $\mathrm{P}$ is located.

$$
2 * m * d P F * \cos \angle P_{F E}=\left(d_{P F}\right)^{2}+\left(d_{E F}\right)^{2}-\left(d_{P E}\right)^{2}
$$

If it does not leave from the rear of the car, then point $P$ has no projection online $\mathrm{AB}$, and there is no formula (1). The left part of formula (1) will be greater than 0 . When the selfsecurity device $\mathrm{P}$ is moved to outside from the rear door of the compartment, as shown in Figure 5, the angle $\angle \mathrm{PFE}$ in triangular PEF is greater than $90^{\circ}$. The left part of formula (1) will be less than 0 . Therefore, in theory, formula (2) can be used as a criterion for the point $\mathrm{P}$ to move out of the rear door of the compartment.

$$
\left(d_{P F}\right)^{2}+\left(d_{E F}\right)^{2}-\left(d_{P E}\right)^{2}<0 .
$$

Cross point $\mathrm{P}$ to make a vertical plane with the planes $A B C D$ and $A D O$, and cross the line $B C$ at point $G$, and cross the line $\mathrm{AD}$ at point $\mathrm{H}$. $\mathrm{PG}$ is perpendicular to $\mathrm{BC}$ in the triangle $\mathrm{PBC} . \mathrm{PH}$ is perpendicular to $\mathrm{AD}$ in the triangle $\mathrm{PAD}$. In triangular $\mathrm{PGH}$, the angles $\angle \mathrm{PGH}$ and $\angle \mathrm{PHG}$ are both less than $90^{\circ}$.

Similar to the above reasons, the criterion for point $\mathrm{P}$ moving out from the front of the compartment is as formula (3), the criterion for point $P$ moving from the left side of the compartment as formula (4), and the criterion for point $\mathrm{P}$ moving out from the right side of the compartment as formula (5).

$$
\begin{aligned}
& \left(d_{P F}\right)^{2}+\left(d_{E F}\right)^{2}-\left(d_{P F}\right)^{2}<0, \\
& \left(d_{P H}\right)^{2}+\left(d_{G H}\right)^{2}-\left(d_{P G}\right)^{2}<0, \\
& \left(d_{P G}\right)^{2}+\left(d_{H G}\right)^{2}-\left(d_{P H}\right)^{2}<0 .
\end{aligned}
$$

In the triangle $\mathrm{APB}$, the area is expressed as follows:

$$
S_{A B P}=\frac{1}{2} d_{A B} d_{P E}=\frac{1}{2} n d_{P E} .
$$




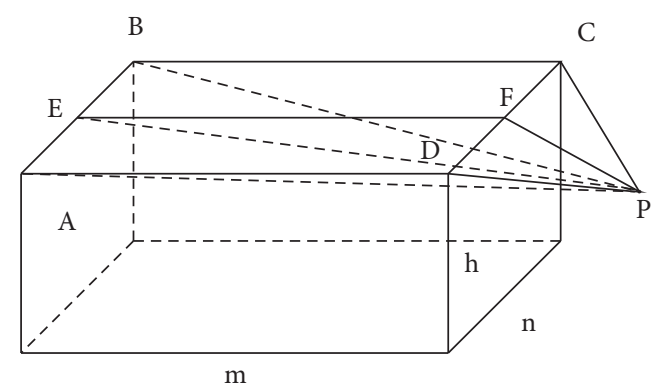

Figure 5: Point $\mathrm{P}$ is located outside, in the backdoor of the compartment.

According to Heron's formula, the area of a triangle APB can also be expressed as follows:

$$
S_{A B P}=\sqrt{L\left(L-d_{A B}\right)\left(L-d_{A P}\right)\left(L-d_{B P}\right)} .
$$

$L$ is half of the circumference, which can be expressed by the following equation:

$$
L=\frac{\left(d_{A B}+d_{A P}+d_{B P}\right)}{2} .
$$

According to formulas (6) and (7), the following formula is obtained:

$$
d_{P E}=\frac{2 \sqrt{L\left(L-d_{A B}\right)\left(L-d_{A P}\right)\left(L-d_{B P}\right)}}{n},
$$

where $d_{P F}, d_{P G}$, and $d_{P H}$ can be obtained similarly. $d_{A P}, d_{B P}$, $d_{C P}$, and $d_{D P}$ can be calculated by wireless signal strength.

\subsection{Illegal Movement Detection Method Based on Space Triangle}

(1) The 4 anchor nodes broadcast frame with their own addresses every $1 \mathrm{~S}$ to provide RSSI. The time interval between the four anchor nodes to send frames is very short, and the time difference can be basically ignored.

(2) According to formula (10), if the noise is filtered out, the distance $d$ can be obtained through the RSSI value [12].

$$
\operatorname{RSSI}_{(k, i)}=A_{k}-10 \eta_{k} \log \left(d_{i k}\right)+v_{(k, i)} .
$$

$R S S I_{(k, i)}$ indicates the wireless signal strength of the data sent by anchor node $i$ received by node k; $A_{k}$ represents the wireless signal strength value one meter away from the anchor node $i ; \eta_{k}$ indicates the environmental attenuation factor; $d_{i k}$ indicates the distance from node $k$ to anchor node i; $v(k, i)$ represents a noise signal with a mean value of 0 .

(3) After filtering the mean value of RSSI data between anchor nodes, $A_{k}$ and $\eta_{k}$ are calculated. The selfsecurity intelligent device is, respectively, placed at a distance of 1 meter from the anchor node $k$ (near the center of the compartment) to actually measure the average value of RSSI. This value is taken as Ak. Along the neutral line of the rear door of the carriage, measure the average RSSI at 10 meters away from the $k$ anchor node, and then derive $\eta_{k}$.

(4) Taking into account the multipath effect, path attenuation, and occlusion effects of the wireless signal in the vehicle compartment, there may be an error in the RSSI value, which generally conforms to the Gaussian distribution. Therefore, it is necessary to perform mean filtering on the RSSI data on the selfsecurity device and then calculate it according to the following formula:

$$
d_{i k}=10\left(\left(A_{k}-R S S I_{(k, i)}\right) / 10 \eta_{k}\right) .
$$

According to formula (11), the distances $\mathrm{d}_{A P}, \mathrm{~d}_{B P}$, $\mathrm{d}_{C P}$, and $\mathrm{d}_{D P}$ can be calculated.

(5) According to formula (9), the distance $d_{\mathrm{PE}}$ can be obtained. In the same way, $d_{P F}, d_{P G}$, and $d_{P H}$ can be obtained.

(6) Calculate the differential of formula (11); we can obtain the following formula:

$\Delta d_{i k}=10\left(\left(A_{k}-\operatorname{RSSI}_{(k, i)}\right) / 10 \eta_{k}\right) *\left(-\frac{1}{10 \eta_{k}}\right) \Delta \operatorname{RSSI}_{(k, i)}$.

Simplifying formula (12), we get the following formula:

$$
\Delta d_{i k}=d_{i k} *\left(-\frac{1}{10 \eta_{k}}\right) \Delta R S S I_{(k, i)} .
$$

According to formula (13), when the RSSI deviation value is constant, the greater the distance $d$, the greater the distance deviation $\Delta \mathrm{d}$. Therefore, the $\mathrm{d}_{P E}$ deviation is greater than $\mathrm{d}_{P F}$, so the right part of formula (1) measurement error $d_{\text {err }}$ should be a negative number. Formulas (2)-(5) are changed to formulas (14)-(17).

$$
\begin{aligned}
& \left(d_{P F}\right)^{2}+\left(d_{E F}\right)^{2}-\left(d_{P E}\right)^{2}<d_{\text {errset }}, \\
& \left(d_{P E}\right)^{2}+\left(d_{E F}\right)^{2}-\left(d_{P F}\right)^{2}<d_{\text {errset }}, \\
& \left(d_{P H}\right)^{2}+\left(d_{G H}\right)^{2}-\left(d_{P G}\right)^{2}<d_{\text {errset }}, \\
& \left(d_{P G}\right)^{2}+\left(d_{H G}\right)^{2}-\left(d_{P H}\right)^{2}<d_{\text {errset }},
\end{aligned}
$$

where $d_{\text {errset }}$ is the error threshold, which is a negative number. Ensure that there is no false alarm in the carriage, and the alarm is required when leaving a certain distance.

3.3. Wireless Signal Strength Data Filtering. When there is less radioactive material in the transport vehicle, the wireless signal is less blocked by obstacles, but the multipath effect 
will be caused by reflection. When there are more radioactive materials in the transport vehicle, the wireless signal is more affected by the obstruction. Multipath effects and obstructions will cause fluctuations in wireless signal strength. This can be seen as noise superimposed on the RSSI value. This noise characteristic generally corresponds to Gaussian noise. Direct use of the raw RSSI data obtained by the measurement will result in larger errors. Therefore, the signal measurement value must be smoothed and preprocessed to eliminate noise.

Here, the mean filtering method is used for filtering. For the 12 RSSI data received from an anchor node, the maximum and minimum values are removed, and the average of the remaining 10 values is calculated. It is shown in the following formula:

$$
\overline{R S S I}=\frac{1}{10} \sum_{i=1}^{10} R S S I_{i} .
$$

where $\overline{R S S I}$ represents the average value of RSSI and $R S S I_{i}$ represents the $i$-th RSSI measurement.

\section{Simulation}

Hongyu brand HYJ5060XFS-JX radioactive material van is shown in Figure 6. The external dimensions are $6350 \times 2120 \times 2800(\mathrm{~mm})$, and the size of the cargo compartment is $4260 \times 2005 \times 1730(\mathrm{~mm})$. The height of the compartment from the ground is $450 \mathrm{~mm}$.

The simulation area is larger than the size of the compartment and is set as $20 \mathrm{~m} \times 1.9 \mathrm{~m}$ (assuming that the antenna is $0.1 \mathrm{~m}$ away from the inner wall of the compartment). It is assumed that the antenna of the self-security device is $1.5 \mathrm{~m}$ away from the top of the compartment. Take the plane where the antenna height is located as the coordinate plane and the lower left corner as the origin to establish a coordinate system. The horizontal and vertical intervals of the grid lines are both 0.5 meters. The entire simulation area is shown in Figure 7. The positions of the four anchor nodes inside the compartment are A $(0.15 \mathrm{~m}$, $0.1 \mathrm{~m}, 1.5 \mathrm{~m}), \mathrm{B}(0.15 \mathrm{~m}, 1.85 \mathrm{~m}, 1.5 \mathrm{~m}), \mathrm{C}(3.45 \mathrm{~m}, 0.1 \mathrm{~m}$, $1.5 \mathrm{~m})$, and $D(3.45 \mathrm{~m}, 1.85 \mathrm{~m}, 1.5 \mathrm{~m})$.

The thief moved the radioactive material container to the back door of the compartment at a speed of $2.25 \mathrm{~m} / \mathrm{s}$. When it was next to the door, the speed slowed down. After getting off the car, he still walked at a speed of $2.25 \mathrm{~m} / \mathrm{S}$. The movement trajectory of the abovementioned radioactive material is indicated by a hollow circle in Figure 8. The solid point represents the $d_{\text {err }}$ value, which is expressed as follows:

$$
d_{e r r}=\left(d_{P F}\right)^{2}+\left(d_{E F}\right)^{2}-\left(d_{P E}\right)^{2} .
$$

It can be seen from Figure 8 that when the radioactive material is inside the compartment, the $d_{\text {err }}$ value is positive. When it moves outside the back door of the compartment, the $d_{e r r}$ value is negative. The $d_{e r r}$ value is monotonically decreasing. So as long as the appropriate $d_{\text {errset }}$ value is set, equation (14) is true. When the $d_{\text {errset }}$ value is set to -5.7956 , and when the radioactive material moves to 14.3 meters from the front of the compartment, that is, 10.1 meters from

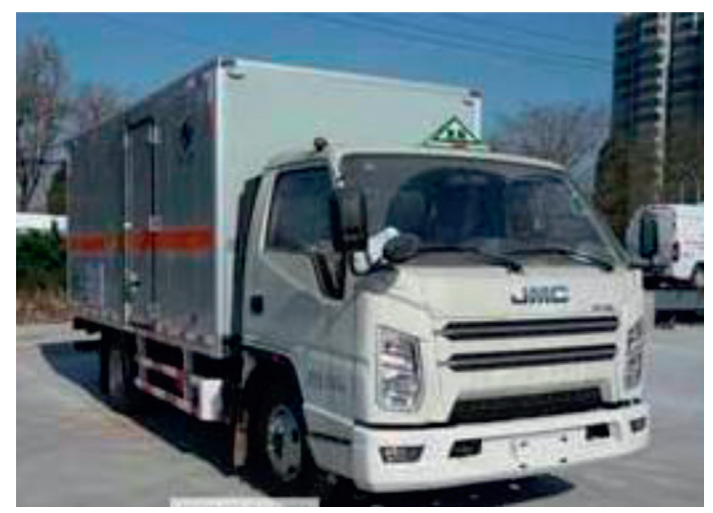

FIGURE 6: HYJ5060XFS-JX radioactive material van.

the compartment, the security system can correctly identify the illegal movement. The radioactive material was found missing when it was far away from the compartment. The reason is that the mean filtering algorithm is adopted and RSSI values are sampled only once every second.

If the thief leaves the compartment at a speed of $0.5 \mathrm{~m} / \mathrm{s}$, the simulation results are shown in Figure 9. When the $d_{\text {errset }}$ value is set to -2.434 , and when the radioactive material moves to 5.3 meters from the front of the compartment, that is, 1.1 meters from the compartment, the security system can correctly identify the illegal movement.

After many simulations, when the radioactive material moves from the inside of the compartment to the outside through the back door of the compartment, the $d_{e r r}$ is monotonously decreasing. So as long as the appropriate $d_{\text {errset }}$ value is set, formula (14) is valid. When radioactive materials are moved from the front and other sides of the compartment, formulas (15), (16), and (17) can also be valid. The farther away from the compartment, the greater the $d_{\text {err }}$ value, so the more sensitive the criterion. It should be noted that when the radioactive material is too far away from the compartment, the wireless signal is too weak. This may cause the self-security device to fail to detect. At the same time, the vehicle security platform will also detect the failure of the communication with the self-security device, and a direct alarm will be issued.

\section{Test Analysis and Discussion}

The experimental system consists of 4 Zigbee terminal nodes as anchor nodes, and one coordinator node as blind node, which is directly connected with the computer. The unauthorized removal detection program and interface based on the space projection angle are realized on the computer. The carriage has only one door, which is located in the rear of the carriage. The internal space dimensions of the carriage are as follows: 3.16 meters in length, 1.74 meters in width, and 1.66 meters in height. After measurement, the height of the bottom of the carriage from the ground is 0.86 meters. During the experiment, the anchor nodes were pasted by transparent glue on the inner wall of the carriage at a height of 1.59 meters, and the layout is shown in Figures 10 and 11. Figure 10 shows the arrangement of anchor nodes at the rear 


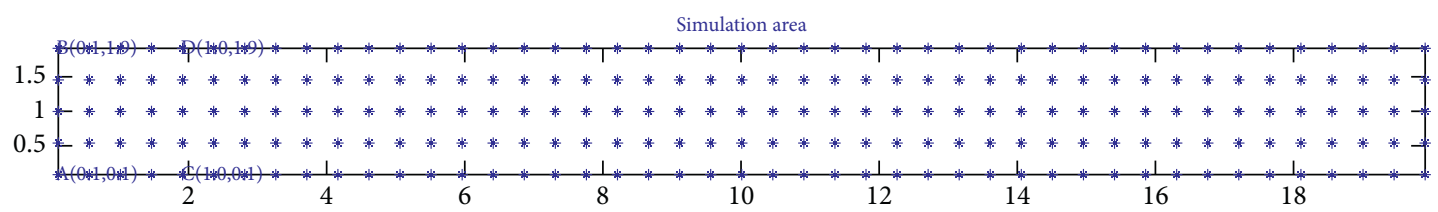

FIgURE 7: The entire simulation area.

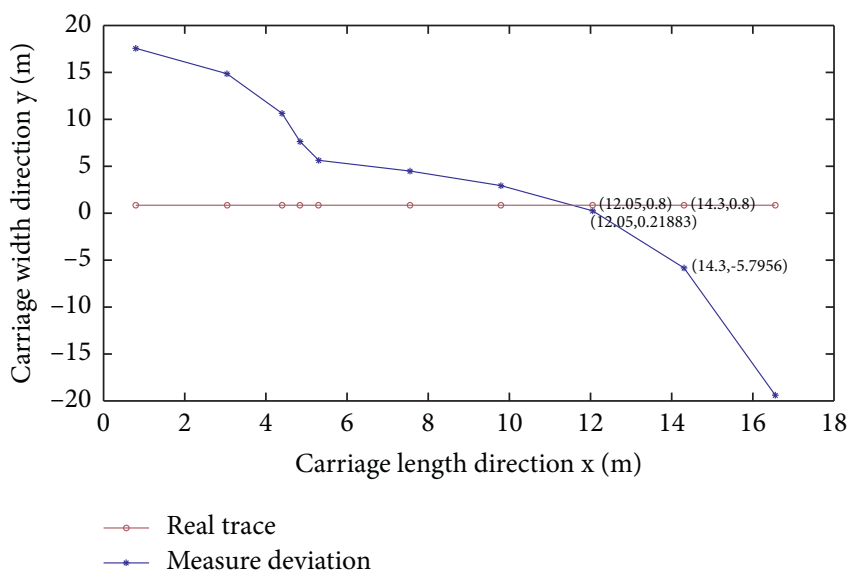

FiguRE 8: Simulation results.

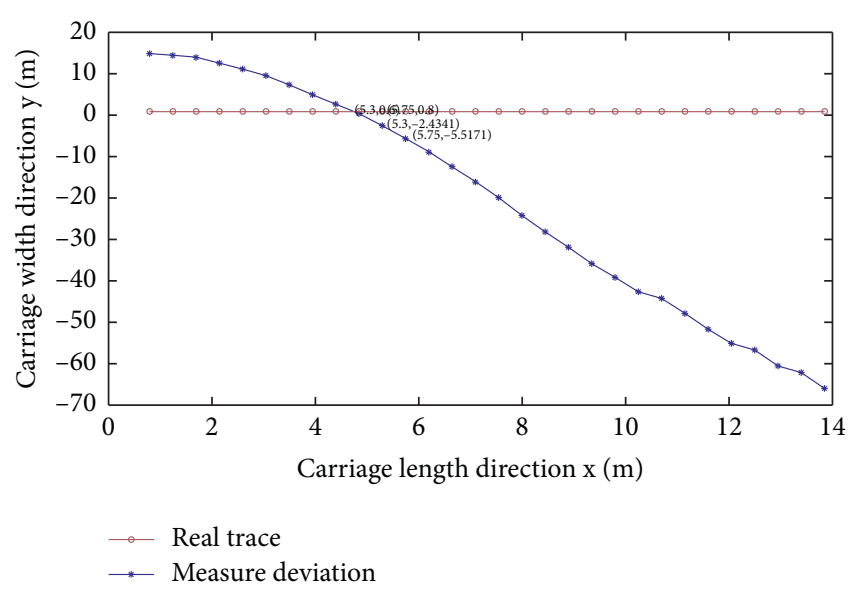

FIGURE 9: Simulation results at $2.25 \mathrm{~m} / \mathrm{s}$ walking speed at $0.5 \mathrm{~m} / \mathrm{s}$ walking speed.

of the carriage, which is 0.15 meters away from the frame. Figure 11 shows the arrangement of the anchor nodes in the front of the carriage, which is also 0.15 meters away from the front of the carriage. The anchor node and the blind node use the same hardware, including Zigbee modules, antennas, buzzers, backplanes, and batteries. In order to facilitate the test, the self-security device is placed in the vehicle compartment 1 meter away from each anchor node, and the measured RSSI is filtered by the mean value as the A value, as shown in Figures 12 and 13. After measurement, the A values of the four anchor nodes are $207 \mathrm{dbm}, 207 \mathrm{dbm}$, $206 \mathrm{dbm}$, and $206 \mathrm{dbm}$, and the $\eta$ values are 2.41, 2.41, 2.43, and 2.43 , respectively.

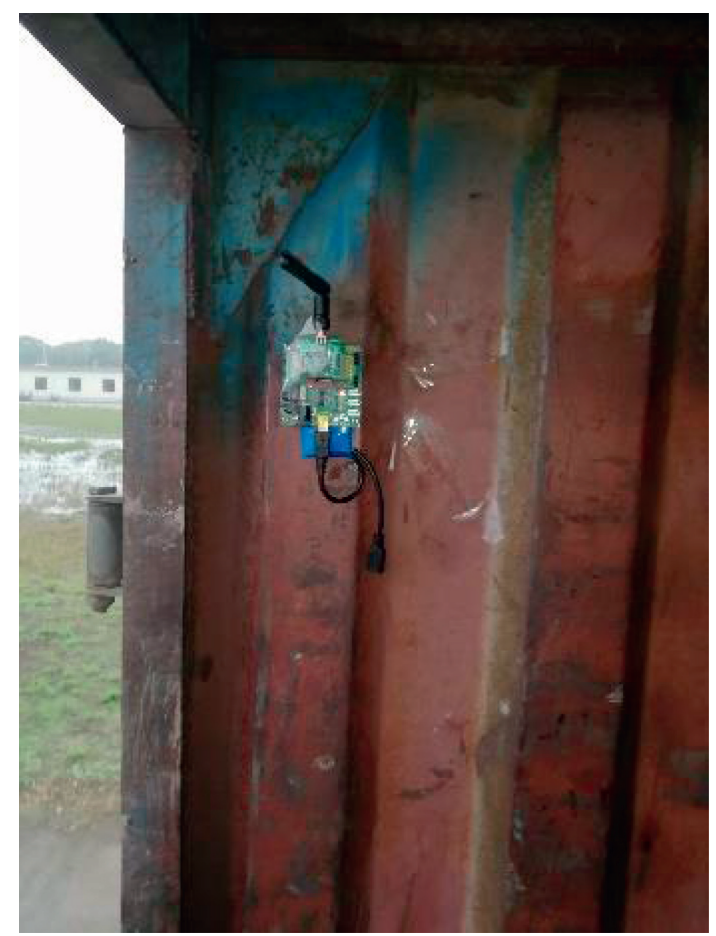

FIGURE 10: Rear anchor node in the carriage.

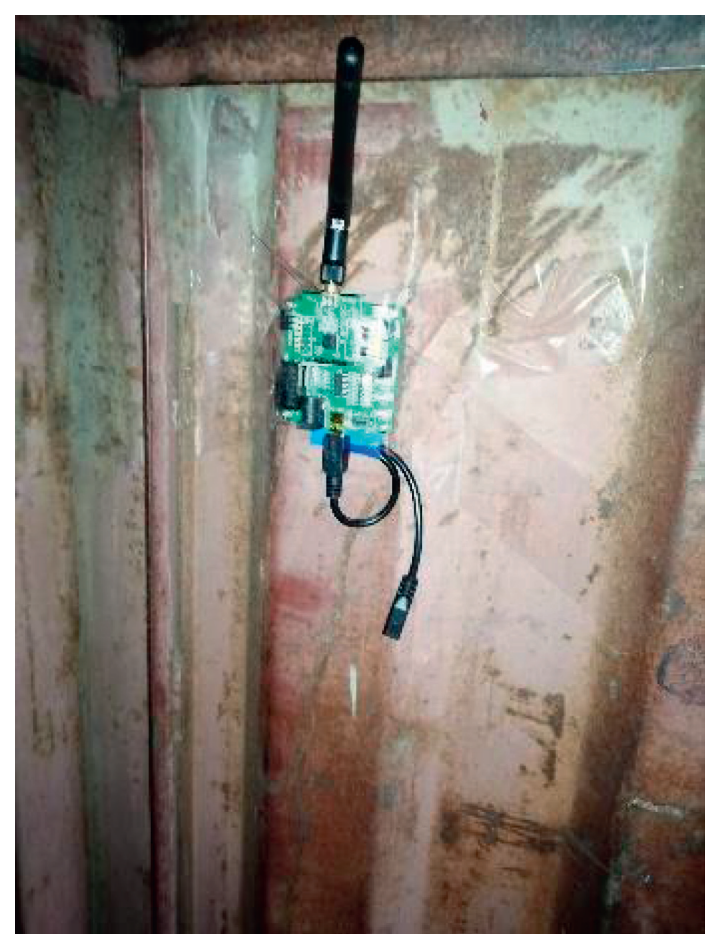

FIGURE 11: Front anchor node in the carriage. 


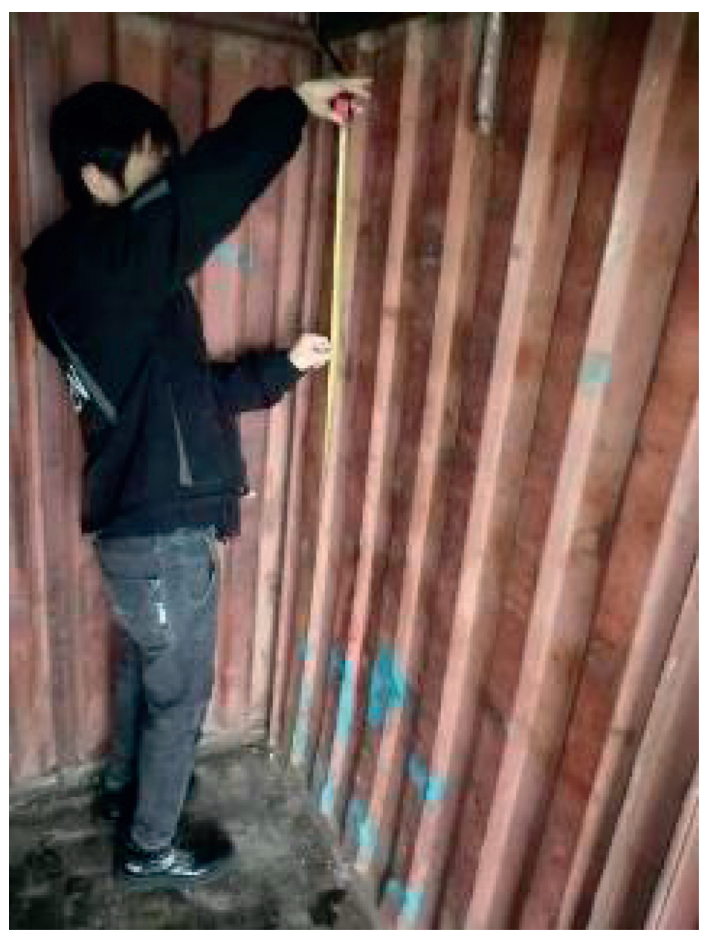

Figure 12: Parameter determination process.

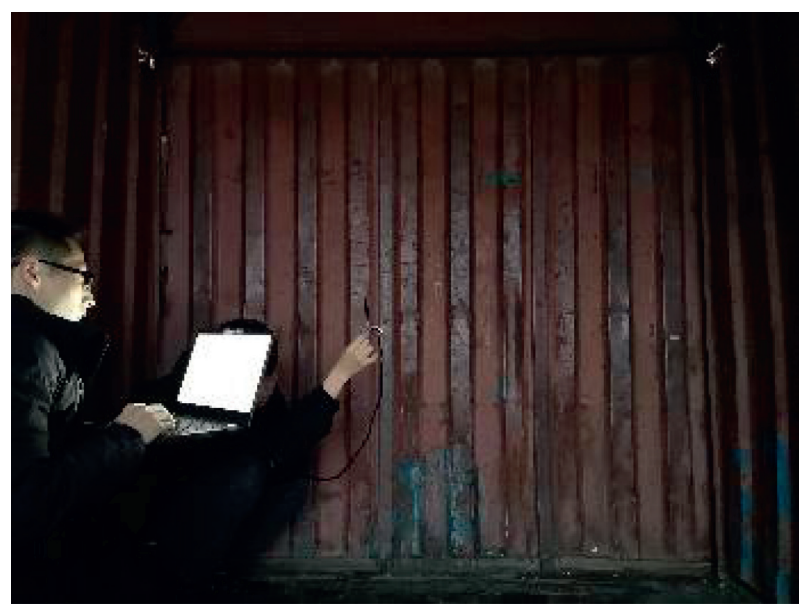

Figure 13: A value measurement process.

The main purpose of our experiment is to verify whether the security system can alarm after the self-security device (blind node) leaves the carriage, and how far away it will alarm. Adjust the threshold to ensure that there is no false alarm in the carriage. After actual measurement, Hderrset, Lderrset, Rderrset, and Bderrset can be selected as -2000 . A person exits from the carriage with a Zigbee coordinator node and stays away (the moving speed is very slow, and the RSSI signal is stable for a period of time after every 0.1 meters of movement). This behavior simulates the loss or theft of the radioactive material container with the electronic equipment from the compartment, as shown in Figure 14. Check the alarm information on the computer, as shown in Figure 15. It is worth pointing out that when the distance

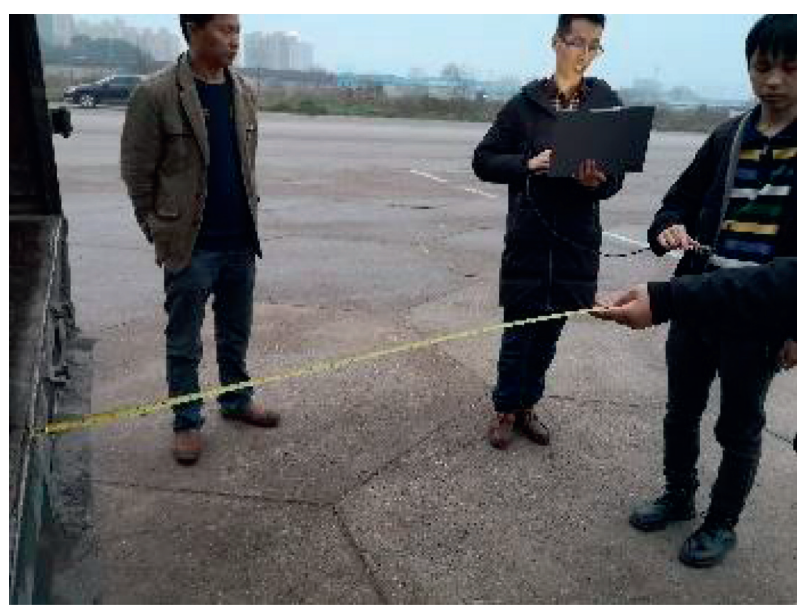

Figure 14: Illegal detection test.

from the left edge of the carriage is less than 0.2 meters, there will be two alarm signals at a certain distance away from the carriage, namely, "illegal movement of the rear" and "illegal movement of the left." There is a similar phenomenon on the right.

Table 2 shows the test data of the security device away from the carriage in three directions: rear, left, and right. Keep away from the center line of the width and length of the carriage, and keep 1.0 meter above the ground when far away, that is, 0.08 meters above the plane of the bottom of the carriage. For example, when moving rear, the width from the left side of the carriage is 0.86 meters. When moving away from the left and right, the length from the rear of the carriage is 1.58 meters. 


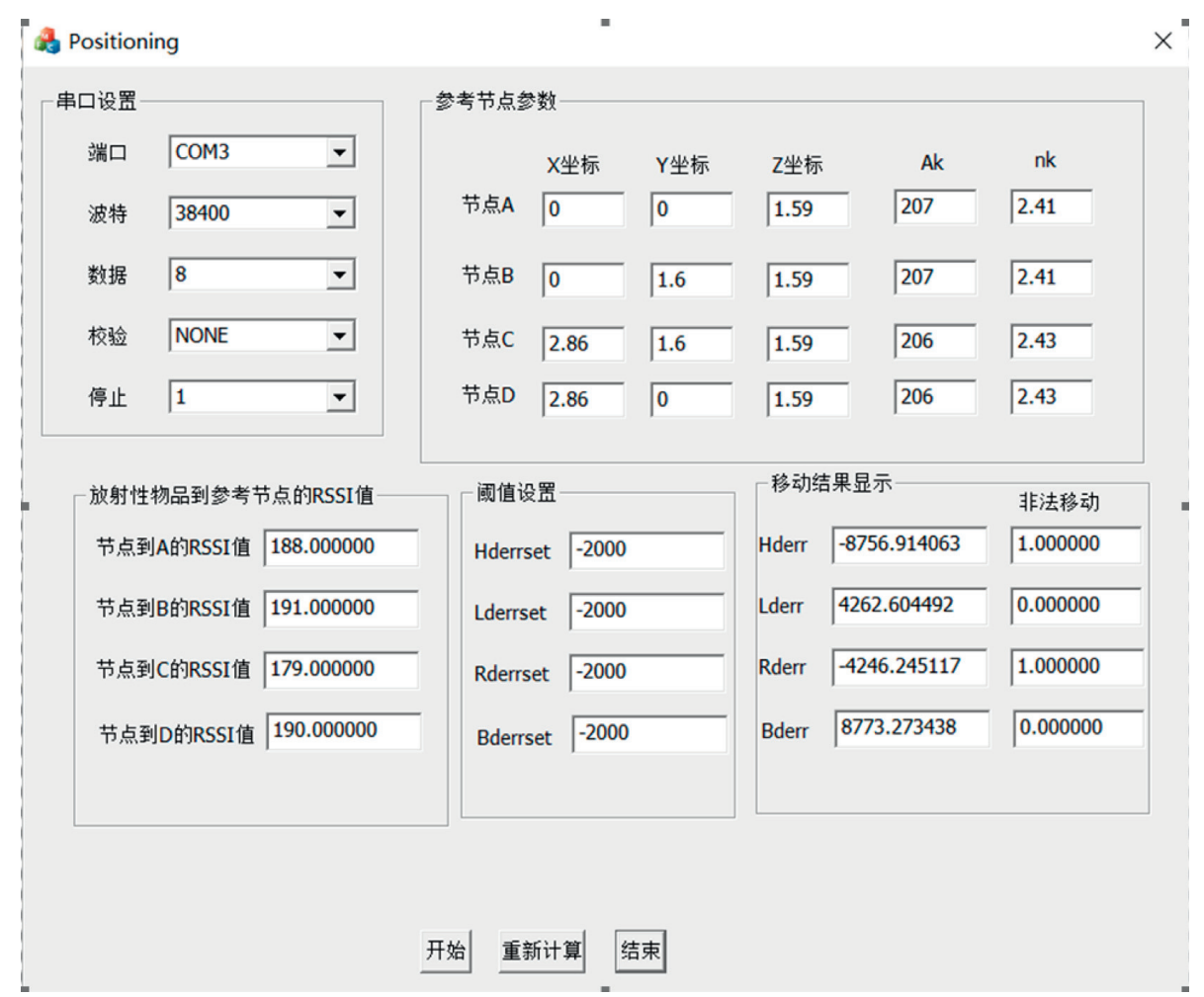

FIGURE 15: Illegal detection test result.

TABLE 2: Illegal detection results based on spatial projection angle.

\begin{tabular}{|c|c|c|c|c|c|c|c|c|c|c|}
\hline & \multicolumn{10}{|c|}{ Alarm distance (unit: meter) } \\
\hline & 1 st & 2nd & 3 rd & 4 th & 5 th & 6th & 7 th & 8th & 9th & 10th \\
\hline Rear of the truck & 2.6 & 2.5 & 2.5 & 3.4 & 2.7 & 2.5 & 2.8 & 3.0 & 2.6 & 3.1 \\
\hline Left of the truck & 1.1 & 1.3 & 1.4 & 1.1 & 1.2 & 1.3 & 1.4 & 1.2 & 1.3 & 1.3 \\
\hline Right of the truck & 1.2 & 1.4 & 1.2 & 1.3 & 1.3 & 1.2 & 1.2 & 1.4 & 1.2 & 1.2 \\
\hline
\end{tabular}

Tests have shown that the farthest distance for the alarm when moving to the rear of the truck is 3.4 meters. The farthest alarm distance of the failure detection method in literature [13] is 26 meters. In comparison, the performance of the method proposed in this paper is better. When changing the $d_{\text {errset }}$ threshold, while maintaining communication, the smaller the $d_{\text {errset }}$ setting (that is, the more negative), the farther the alarm distance will be, but the higher the reliability. It will also alarm when it is far away from the left and right, and when it is very close to the carriage. This is because the iron sheet material of the carriage shields the signal. When away from the front, the security system will directly alarm. Because the enclosed compartment shields the wireless signal, the front of the vehicle cannot receive the wireless signal.

\section{Conclusions}

In view of the different characteristics of normal movement and illegal movement during the transportation of radioactive materials, the movement to the outside of the compartment is proposed as the detection standard for "illegal movement." Four anchor nodes are installed in the compartment, and the self-security device receives wireless signals. The wireless signal strength data is filtered and converted into distance data. Based on the distance data, a spatial triangle is constructed, and an "illegal movement" sensing method is proposed. The simulation and field test show that the method proposed in this paper is feasible.

\section{Data Availability}

The data used to support the findings of this study are included within the article.

\section{Conflicts of Interest}

The authors declare that there are no conflicts of interest regarding this publication of this study.

\section{Acknowledgments}

This study was funded by China's 13th Five-Year Nuclear Energy Development Project "Individual Self-Security Intelligence of Radioactive Material.” 


\section{References}

[1] T. Zeng, X. Yang, Y. Wan, M. Yu, and L. Zhenghai, "Vehicle transport security system based on the self-security intelligence of radioactive material," Science and Technology of Nuclear Installations, vol. 2020, Article ID 3507167, 7 pages, 2020.

[2] Y. Pan, F. Cao, and Z. Hong, "The study of building national online monitoring platform for transport of category I and II radioactive material," Progress Report on China Nuclear Science \& Technology, vol. 5, pp. 351-360, 2017, in Chinese.

[3] https://www.nrc.gov/reading-rm/doc-collections/event-status/ event/2020/20201102en.html.

[4] IAEA, NSS 9-G(Rev. 1): Security of Radioactive Material in Transport, IAEA, Vienna, Austria, 2020.

[5] X. Yang, T. Zeng, and Y. Wan, "Self-security intelligence of individual radioactive substance," Nuclear Safety, vol. 18, no. 02, pp. 43-48, 2019, in Chinese.

[6] X. Yang, J. Hu, Y. Wan, Z. Liu, and Y. Mao, "Swarm selfsecurity intelligence of radioactive substances," Nuclear Safety, vol. 6, pp. 1-5, 2019.

[7] T. Zeng, X. Yang, Y. Wan, L. Zhenghai, and J. Panpan, "Single-vehicle transportation security system of radioactive materials based on group relationship to prevent loss and theft," Science and Technology of Nuclear Installations, vol. 2021, Article ID 6649649, 9 pages, 2021.

[8] Anonymous, Regulations on Road Transportation of Radioactive Materials, China Road Transport, China, revised edition, 2016.

[9] https://www.ne.anl.gov/packaging-certification-and-life-cycle -management/argus-rfid.

[10] Z. Zhang, Z. Liu, and R. Zhang, " $\gamma$ research on radiation source falling off early warning and $\mathrm{Nb}$ IOT real-time monitoring system of flaw detector," New industrialization, vol. 009, no. 010, pp. 5-12, 2019.

[11] H. Pujiharsono, D. Utami, and R. D. Ainul, "Trilateration method for estimating location in RSSI-based indoor positioning system using zigbee protocol," Jurnal Infotel, vol. 12, no. 1, pp. 1-6, 2020.

[12] C. Zhou, H. Tian, and B. Zhong, "An improved MCB localization algorithm based on weighted RSSI and motion prediction," Computer Science and Information Systems, vol. 20, no. 00, 2020.

[13] Z. Zhang, Indoor Positioning Based on RSSI Ranging, Anhui University of Technology, Ma'anshan, China, 2015. 